\title{
Gestión de riesgos por eventos extremos de precipitación: el caso de los funcionarios municipales del estado de Veracruz
}

Recibido: 31/01/2016 · Aceptado: 05/04/2016

\author{
Carolina A. Ochoa-Martínez* \\ Centro de Ciencias de la Tierra, Universidad Veracruzana \\ Ana Cecilia Travieso-Bello \\ Facultad de Economía, Universidad Veracruzana \\ Carlos Manuel Welsh-Rodríguez \\ Centro de Ciencias de la Tierra, Universidad Veracruzana \\ Cecilia Conde-Álvarez \\ Centro de Ciencias de la Atmósfera, Universidad Nacional Autónoma de México \\ Gladis Yáñez-Garrido \\ Doctorado en Ecología Tropical, Universidad Veracruzana
}

\section{Resumen}

El estudio de la gestión de riesgos ante los eventos hidrometeorológicos extremos es un componente fundamental para que los sistemas de alerta temprana puedan resultar instrumentos de respuesta efectiva. En este artículo se analiza la gestión del riesgo por eventos extremos de precipitación por parte de los funcionarios municipales del estado de Veracruz y se comparan las regiones norte, centro y sur de la entidad. Se aplicó un cuestionario en materia de protección civil a funcionarios de 58 municipios del estado de Veracruz, que representan $27 \%$ del total y abarcan $40 \%$ del territorio veracruzano. Se obtuvo información sobre su grado de escolaridad, conocimiento de su función, así como sus formas de actuación antes, durante y después de la presencia de eventos extremos de precipitación. Los resultados mostraron que el máximo grado de estudios de la mayoría de los entrevistados fue maestría (38\%). Las organizaciones actúan antes, durante y después de un evento extremo de precipitación con 73 \% para la zona norte y $36 \%$ para las zonas centro y sur. Por último, se observó que los gobiernos municipales por lo general se limitan a la emisión de alertas meteorológicas y a las actividades relacionadas con albergues. Se recomienda la capacitación y la comunicación como estrategias esenciales para reducir la vulnerabilidad y aumentar la resiliencia en materia de cambio climático.

Palabras clave: Eventos extremos, cambio climático, gobiernos municipales, Veracruz.

*Correo electrónico: caochoa@uv.mx 


\title{
Risk management in extreme precipitation events by municipal authorities in the state of Veracruz
}

\author{
Carolina A. Ochoa-Martínez* \\ Centro de Ciencias de la Tierra, Universidad Veracruzana \\ Ana Cecilia Travieso-Bello \\ Facultad de Economía, Universidad Veracruzana \\ Carlos Manuel Welsh-Rodríguez \\ Centro de Ciencias de la Tierra, Universidad Veracruzana \\ Cecilia Conde-Álvarez \\ Centro de Ciencias de la Atmósfera, Universidad Nacional Autónoma de México \\ Gladis Yáñez-Garrido \\ Doctorado en Ecología Tropical, Universidad Veracruzana
}

\begin{abstract}
Studying risk management in extreme hydro-meteorological events constitutes a fundamental element for early warning systems to be effective response instruments. This article analyzes risk management by municipal authorities in the case of extreme rainfall in the state of Veracruz comparing the state's northern, southern and central regions. A survey on public safety issues was applied to local authorities of 58 municipalities, which represent $27 \%$ of the total and cover $40 \%$ of the state territory. Information on their education level, knowledge of their work responsibilities and their performance prior to, during and after extreme precipitation events was generated. Results show that the maximum educational level of the majority of interviewees (38\%) was a master degree. When facing extreme precipitation events, the level of response of organizations in the northern zone is $73 \%$ while in the central and southern zones it is $36 \%$. In general, municipal governments limit their actions to the broadcasting of meteorological alerts and shelter related activities. It is recommended basic strategies on communications and capacitation in order to reduce vulnerability and increase resilience to climate change. Training and communication are recommended as essential strategies to reduce vulnerability and to increase resilience in climate change issues.
\end{abstract}

Keywords: Extreme events, climate change, local governments, Veracruz.

*Correo electrónico: caochoa@uv.mx 


\section{Introducción}

El cambio climático es un tema que preocupa de manera creciente a investigadores, científicos y gobiernos. El Panel Intergubernamental de Cambio ClimátiCo (IPCC, por sus siglas en inglés), en su V Reporte de Evaluación, ha señalado que el calentamiento del sistema climático es inequívoco; constituye un fenómeno que se observa en los incrementos de los promedios globales de temperatura del aire y de los océanos, así como en la disminución de la cubierta de nieve del planeta y en la elevación del nivel del mar (IPCC, 2014, p. 12). Por tanto, se ha convertido en una realidad para todos los habitantes del planeta y en una problemática vinculada con el desarrollo y la seguridad de cualquier nación.

Los efectos del cambio climático, en particular los relacionados con los eventos meteorológicos extremos, están aumentando su grado de incidencia en las comunidades y asentamientos humanos. Por ello, en la conferencia de las partes (COP21) se estableció el compromiso internacional de promover la observación sistemática del clima, además de fortalecer los sistemas nacionales de alerta temprana, como acciones esenciales a desarrollar para la adaptación humana al cambio climático. Esto quedó plasmado en el documento final denominado Acuerdo de París (Naciones Unidas, 2015).

En consecuencia, se ha vuelto prioritario incluir en el desarrollo local y regional programas de detección de riesgos y de vulnerabilidades sociales, así como la preparación de acciones de prevención de desastres y de respuestas efectivas de protección civil. Estos temas se han convertido en un asunto de preocupación no solo para la comunidad científica, sino para los encargados de definir y conducir las políticas públicas nacionales e internacionales, incluyendo a los tomadores de decisiones en los distintos niveles y ámbitos de gobierno relacionados con esta problemática.

En México, las políticas para investigar y atender los problemas asociados al cambio climático y sus consecuencias han sido diseñadas y establecidas a escala nacional y desde la perspectiva federal. Sin embargo, el conocimiento específico de la forma en que estos fenómenos ocurren e impactan a la población se encuentra en las entidades federativas, en los municipios y en sus localidades, lo que contrasta con la limitada capacidad de reacción, ante estos problemas, por parte de los gobiernos estatales, y especialmente de los municipales (Welsh et al., 2015). 
Los gobiernos municipales tienen un papel central en el desarrollo y el bienestar social del país. El artículo 115 de la Constitución Política de los Estados Unidos Mexicanos (Cámara de Diputados, 2016) señala las funciones y servicios que deben proporcionar los municipios, entre ellos el suministro de agua potable, drenaje y alcantarillado; el tratamiento y disposición de aguas residuales; la participación en la creación de reservas ecológicas, y el establecimiento de programas de ordenamiento territorial y urbano, principalmente. A estos se agrega informar y sensibilizar a las comunidades a fin de prevenir riesgos, así como diseñar y desarrollar políticas públicas y acciones de respuesta para el amortiguamiento de los efectos locales o regionales derivados de las variaciones climáticas o eventos hidrometeorológicos extremos.

Se reconoce que los gobiernos municipales desempeñan un rol esencial en la aplicación adecuada de instrumentos de planeación territorial y gestión de riesgo. El nuevo Sistema de Alerta Temprana para Ciclones Tropicales (Segob, 2015) establece que los municipios instalarán centros de coordinación y comunicación para prevenir y atender la inminencia de un desastre hidrometeorológico, y que sus integrantes formarán parte del Sistema Nacional de Protección Civil. Por ello se ha considerado una tarea urgente fortalecer las capacidades de este nivel de gobierno (Semarnat, 2012, p. 121).

La gestión del riesgo es un concepto clave en la atención y prevención de desastres. La Ley General de Protección Civil, en su artículo $2^{\circ}$ fracción xxviI, define la gestión integral de riesgos (GIR) como:

El conjunto de acciones encaminadas a la identificación, análisis, evaluación, control y reducción de los riesgos, considerándolos por su origen multifactorial y en un proceso permanente de construcción, que involucra a los tres niveles de gobierno, así como a los sectores de la sociedad, lo que facilita la realización de acciones dirigidas a la creación e implementación de políticas públicas, estrategias y procedimientos integrados al logro de pautas de desarrollo sostenible, que combatan las causas estructurales de los desastres y fortalezcan las capacidades de resiliencia o resistencia de la sociedad. Involucra las etapas de: identificación de los riesgos y/o su proceso de formación, previsión, prevención, mitigación, preparación, auxilio, recuperación y reconstrucción [Cámara de Diputados, 2012, s. p.]. 
Para dar un mejor panorama, el riesgo es descrito en el mismo artículo (fracción xLVIII) como los "daños o pérdidas probables sobre un agente afectable, resultado de la interacción entre su vulnerabilidad y la presencia de un agente perturbador" (Cámara de Diputados, 2012, s. p.). Diversos autores lo señalan como una medida de la probabilidad de que ocurran efectos adversos. Implica la percepción del riesgo por parte de individuos o grupos sociales, es decir, la habilidad individual o colectiva para discernir el grado de riesgo (real o esperado), y la capacidad de tolerancia y vulnerabilidad a él (Renn, 1998; Inouye, 2014).

Por otra parte, el Sistema de Alerta Temprana (SAT) se define como el conjunto de procedimientos e instrumentos a través de los cuales se monitorea una amenaza o evento adverso (natural o antrópico) de carácter previsible, se recolectan y procesan datos e información, ofreciendo pronósticos o predicciones temporales sobre su acción y posibles efectos (Armién, 2012). En ese orden de ideas, se considera que la gestión del riesgo por parte de los funcionarios municipales encargados de los sistemas de alerta temprana y protección civil representa un aspecto importante en la tarea de prevenir y reducir el impacto local de los riesgos climáticos actuales y futuros.

De acuerdo con el IPCC (2012, p. 10), se carece de un inventario de desastres en el plano local, lo que limita el mejoramiento de los procesos para reducir la vulnerabilidad. Sin embargo, existen desigualdades a escala local en el modo de enfrentar un desastre, que influyen en la capacidad de adaptación. Asociado a lo anterior, se encuentra el nivel de preparación y las formas de actuación de los funcionarios públicos antes, durante y después de un evento meteorológico extremo.

En este trabajo se analiza la gestión del riesgo por eventos extremos de precipitación por parte de los funcionarios municipales del estado de Veracruz y se comparan las regiones norte, centro y sur de la entidad.

\section{Zona de estudio}

El estado de Veracruz representa $3.7 \%$ de la república mexicana, con una superficie de $71820.4 \mathrm{~km}^{2}$ y una franja costera de $684 \mathrm{~km}$. Ocupa el tercer lugar nacional en población, el cuarto en producción agrícola y aporta $5.2 \%$ al producto interno bruto del país, ubicándose en el quinto lugar nacional (INEGI, 
2012). Además, se encuentra entre las siete entidades federativas más vulnerables al cambio climático (Monterroso et al., 2014).

El escenario climático del estado es extraordinariamente diverso. Los climas varían desde el cálido húmedo en el sur, hasta el cálido semiseco en el norte, pasando por las condiciones templadas y frías de la montaña (Ruiz Barradas, Tejeda Martínez, Miranda Alonso y Flores Zamudio, 2010, p. 67). Asimismo, debido a su localización geográfica y alta biodiversidad, recibe impactos meteorológicos que afectan los sectores productivos y social (Vázquez Torres, Carvajal Hernández y Aquino Zapata, 2010, p. 251).

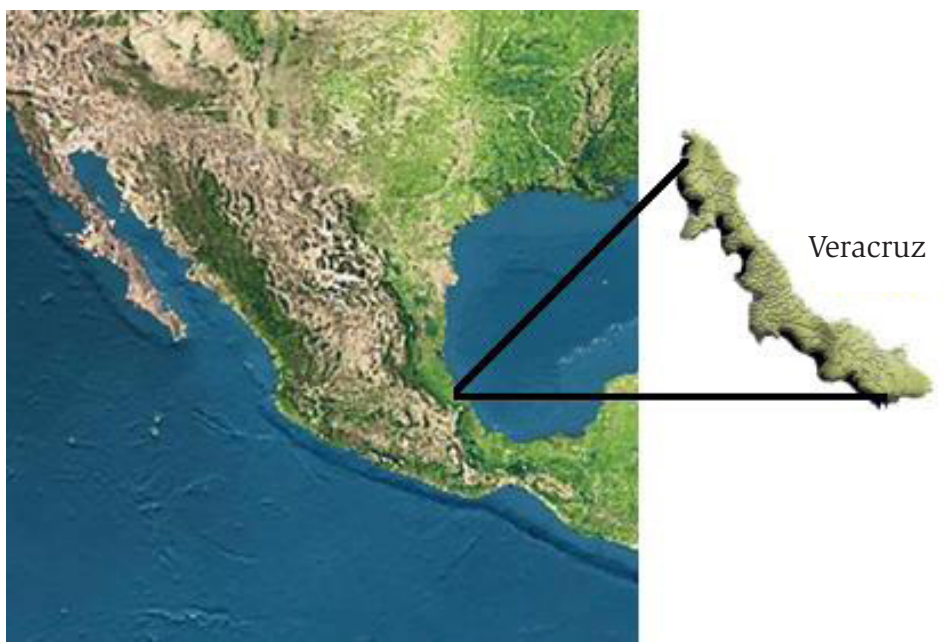

Fuente: Tejeda-Martínez et al. (2008).

Figura 1. Ubicación geográfica del estado de Veracruz

Por su ubicación respecto a la región tropical y al Golfo de México, la entidad veracruzana está expuesta al paso de los ciclones tropicales. Estos eventos generalmente ocurren de mayo a noviembre (Palma, Morales y Díaz, 2012, p. 70). Sin embargo, inciden con mayor frecuencia en septiembre y agosto. Cabe señalar que aunque estos no penetren a tierra, pueden causar daños considerables en cualquiera de sus etapas de desarrollo (Travieso-Bello, 2012, p. 90). Por otra parte, los "nortes" se presentan sobre todo de octubre a mayo y pueden 
ocasionar precipitaciones sobre las cordilleras al este de México y potencialmente producir inundaciones en el estado (Acevedo y Luna, 2006).

En el periodo invernal, los frentes fríos azotan con vientos superiores a los $70 \mathrm{~km} / \mathrm{h}$, en promedio durante 35 días al año. Esto provoca descensos de temperatura superiores a $10{ }^{\circ} \mathrm{C}$ en 24 horas. En contraste, durante el verano, el aire tropical, a veces en forma de perturbación o ciclón, provoca inundaciones en las partes bajas y deslaves en las sierras (Ruiz Barradas et al., 2010, p. 65).

En México, los desastres asociados a eventos climáticos extremos han tenido un importante incremento en los últimos años. En el periodo 2000-2013 se emitieron 7231 declaratorias, de las cuales $92.5 \%$ concernió a eventos hidrometeorológicos (ciclones tropicales, fuertes vientos, inundaciones, lluvias, nevadas, heladas, granizadas, sequías y tornados). Destacan los ciclones tropicales e inundaciones, que representan $81 \%$ del total de los eventos hidrometeorológicos. En particular, $27 \%$ de ellos ocurrieron en el estado de Veracruz y corresponden a inundaciones (49\%), precipitaciones extremas (28\%) y huracanes (20\%) (Ochoa, Ibarra, Welsh y Yáñez, 2015).

Cabe resaltar que Veracruz es pionero en materia de cambio climático, ya que fue el primer estado en la república mexicana que elaboró el Plan Estatal ante el Cambio Climático, el cual sirvió de guía para el resto del país. Empero, la incidencia de huracanes y tormentas tropicales ha aumentado su frecuencia en los últimos años: en el 2000 fue el huracán Keith; en 2005, Emily y Stan; en 2007, Dean y Lorenzo; en 2008, la tormenta tropical Marco, mientras que en 2010, el huracán Karl e inmediatamente después la tormenta tropical Matthew.

A escala mundial se espera que la frecuencia de precipitaciones intensas o la proporción de lluvias totales derivadas de precipitaciones intensas se incremente en muchas zonas del planeta durante el siglo XXI (IPCC, 2012, p. 12).

Por otro lado, se prevé que para el estado de Veracruz existan variaciones en la precipitación total anual de +5 a $-5 \%$ para 2020 y de +10 y $-20 \%$ para 2080. Asimismo, la temperatura se elevará entre 0.8 y $1.4{ }^{\circ} \mathrm{C}$ y entre 2 y $3{ }^{\circ} \mathrm{C}$ para 2020 y 2080, respectivamente (InECC, 2016).

\section{Metodología}

100 Para la obtención de los datos de análisis del presente estudio se consideró un universo compuesto por los funcionarios municipales de las zonas norte, 
centro y sur del estado de Veracruz que asistieron a los Talleres de Inducción a los Programas de Acción Climática Municipal. Esta división por zonas responde a las características del territorio veracruzano, que se extiende en una franja a lo largo de la costa del Golfo de México. Además, en términos de logística, fue más fácil encuestar a los funcionarios que se agruparon por zona para tomar los talleres. Estos se impartieron en el nivel nacional de 2013-2015 y en ellos participaron aproximadamente 250 municipios del país, los cuales representaron poco más de $10 \%$ del total nacional; entre ellos figuraron 70 municipios del estado de Veracruz.

En esa entidad, la Secretaría de Medio Ambiente del Gobierno del Estado, a través de la Unidad de Cambio Climático, fue la encargada de coordinar un taller por zona, que fueron impartidos por la Universidad Veracruzana, El Colegio de Veracruz, el Instituto de Ecología, A. C., Gobiernos Locales por la Sustentabilidad (ICLeI), la Secretaría de Salud y la Secretaría de Medio Ambiente.

\begin{tabular}{|l|ll} 
Canderilla \\
Boca del Rio \\
Camerino Z. Mendoza \\
Carrillo Puerto \\
Coatepec \\
Córdoba
\end{tabular}

Fuente: Elaboración propia.

FiguRa 2. Distribución geográfica de los municipios participantes en el estudio 
Se diseñó un cuestionario dirigido a conocer edad, escolaridad, tiempo de residencia en la zona y en el puesto, así como las formas de actuación y de respuesta de los funcionarios municipales antes, durante y después de los eventos extremos de precipitación en el estado de Veracruz. Este se aplicó a 105 funcionarios de 58 municipios (figura 2), que representan $27 \%$ del total de los $212 \mathrm{mu}$ nicipios del estado, y que abarcan 40 \% del territorio veracruzano. La aplicación del cuestionario se realizó durante la segunda etapa del taller, después de que los asistentes habían recibido capacitación sobre conceptos generales del tema.

\section{Resultados y discusión}

A efectos de atender la gestión integral del riesgo es necesario tener conocimientos disciplinares y especializados que permitan aproximarse con una base académica, razón por la cual es importante conocer si quienes ostentan una responsabilidad pública en este ámbito cuentan con estudios de pregrado y posgrado. Se encontró que el grado máximo de estudios que tiene la mayoría de los funcionarios encuestados fue de maestría (38\%); se trata de personas que no solo poseen conocimientos disciplinares, sino que son especialistas en un área del conocimiento y que con certeza pueden apropiarse y trabajar en esta temática. Se hallaron diferencias entre zonas: en la norte, el grado máximo fue de licenciatura (70 \%), mientras que en la centro y sur, el grado de maestría alcanzó 45 y $68 \%$, respectivamente.

El conocimiento de la zona de influencia no se basa únicamente en haber nacido en una región determinada o haber permanecido allí por mucho tiempo, sin embargo, quienes mejor conocen un espacio, región o territorio son las personas que han permanecido en ellos por más tiempo. En ese sentido, los funcionarios de la zona norte tienen más de 40 años de residencia ahí (40\%), mientras que los del centro solo han residido en la zona entre uno y cuatro años (23 \%); respecto al sur, existe una igualdad de $29 \%$ en las categorías de 20 a 29 y de 30 a 39 años de residencia en la zona. Por tanto, se infiere que los funcionarios de las zonas norte y sur tienen mayor conocimiento de su territorio. En contraste, es probable que los funcionarios de la zona centro lleguen de otros sitios de residencia para desempeñar su función. 
Es relevante apuntar que el grado jerárquico de cargo más frecuente de los entrevistados fue "jefe de departamento" o "director de área”, con 60, 38 y $36 \%$ para las zonas norte, centro y sur, respectivamente. Por tanto, son funcionarios del gobierno municipal capaces de incidir en la gestión integral del riesgo, en el ámbito de la toma de decisiones ante un evento extremo. Por ello se les cuestionó acerca del tiempo que han estado en la organización, a lo que $63 \%$ de los entrevistados en la zona norte mencionó que de uno a tres años, en tanto, en las zonas centro y sur han estado menos de un año (47 y $54 \%$ ). Esto puede reflejar que se trata de un periodo de transición de la administración o, peor aún, que son áreas consideradas de poca importancia y en las que no es necesaria la construcción de capacidades para el servicio civil de carrera. En términos de las capacidades institucionales es evidente que, durante las últimas décadas, México ha ido creando y reforzando algunas instituciones para mejorar de manera significativa la gestión de recursos naturales y el diseño de políticas para atender la mitigación y adaptación al cambio climático. No obstante, alcanzar y mantener estas capacidades institucionales requiere de fuertes inversiones presupuestales, así como la formación de recursos humanos especializados (Fernández Bremauntz, 2012). Esto último es necesario para atender lo establecido en la Ley General de Protección Civil (véase cuadro 1).

\section{CUADRO 1. Respuesta mayoritaria para cada zona de estudio}

\begin{tabular}{|c|c|c|c|}
\hline \multirow{2}{*}{ VARIABLES } & \multicolumn{3}{|c|}{ Zona } \\
\hline & Norte & Centro & Sur \\
\hline $\begin{array}{l}\text { Número de municipios } \\
\text { donde se aplicaron } \\
\text { entrevistas }\end{array}$ & 30 & 16 & 12 \\
\hline Edad ( > 30 años) & $67 \%$ & $66 \%$ & $63 \%$ \\
\hline $\begin{array}{l}\text { Nivel máximo de } \\
\text { estudio }\end{array}$ & $\begin{array}{l}\text { Licenciatura } \\
\quad(70 \%)\end{array}$ & $\begin{array}{c}\text { Maestría } \\
(45 \%)\end{array}$ & $\begin{array}{c}\text { Maestría } \\
(68 \%)\end{array}$ \\
\hline Tiempo de residencia & $\begin{array}{c}> \\
\quad 40 \text { años } \\
(40 \%)\end{array}$ & $\begin{array}{c}\text { De } 1 \text { a } 4 \text { años } \\
(23 \%)\end{array}$ & $\begin{array}{c}\text { De } 20-29 \text { y } \\
\text { de } 30-39 \text { años }(29 \%)\end{array}$ \\
\hline $\begin{array}{l}\text { Tiempo de permanencia } \\
\text { en la organización }\end{array}$ & 1 a 3 años (63\%) & $<1$ año (47\%) & $<1$ año (54\%) \\
\hline
\end{tabular}

Fuente: Elaboración propia.

El conocimiento del tiempo de residencia del funcionario entrevistado permite asumir que conoce el clima local de la zona donde trabaja, así como los fenómenos hidrometeorológicos a los que se enfrenta, ya que dentro de sus 
actividades o servicios están las alertas meteorológicas como respuesta principal, además de la elaboración de programas ante tales fenómenos, lo que señala una cultura de la prevención. Sin embargo, el corto tiempo de permanencia en la organización indica la transición de personal que sufren los municipios en cada gestión, lo cual podría dificultar la continuidad del trabajo de prevención que se esté realizando, sobre todo si este personal no tiene un buen conocimiento de la variabilidad estacional y de cómo responde la población a los fenómenos meteorológicos. Por lo tanto, se puede afirmar que es necesario establecer, de forma específica, relaciones de vinculación reales que permitan fortalecer el trabajo en el sector municipal.

De igual modo se les cuestionó si pensaban que ha aumentado la cadena en la toma de decisiones: en la zona norte $63 \%$ dijo no saber

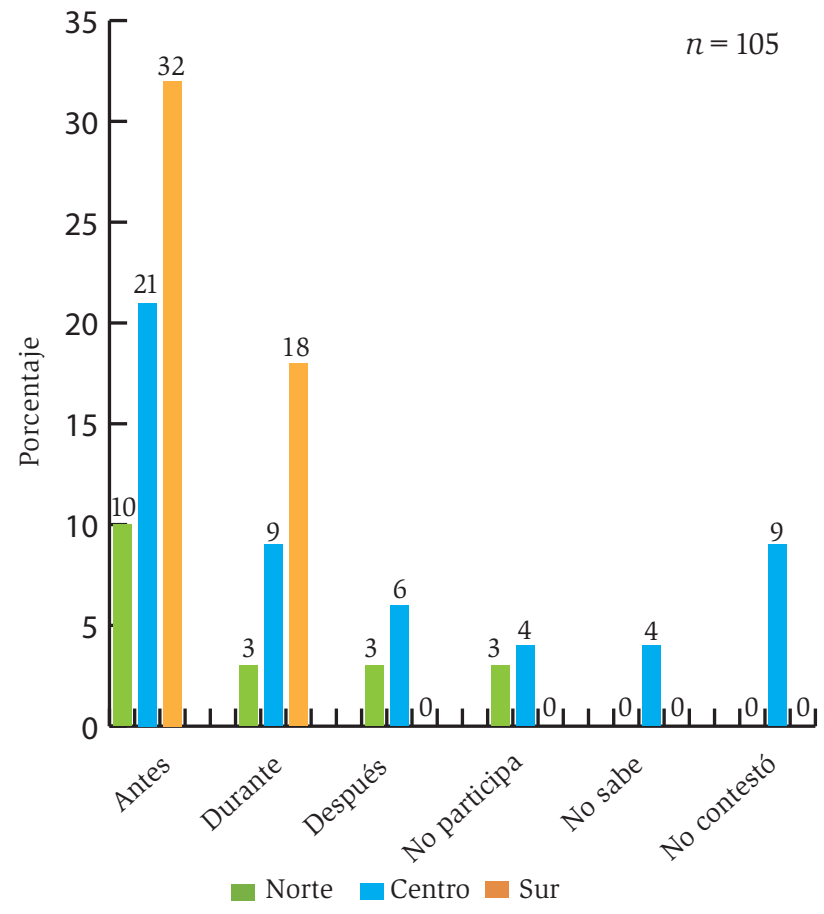

Fuente: Elaboración propia.

Figura 3. Porcentaje de respuestas a la pregunta: ¿Está su organización involucrada en la preparación o la respuesta a los eventos extremos de precipitación? 
(siendo los que presentaron mayor permanencia en el puesto y en la comunidad), mientras que en las zonas centro y sur sí consideran un aumento: 55 y $64 \%$, respectivamente. Es decir, que uno de cada dos funcionarios considera un aumento en la cadena de toma de decisiones ante un evento de precipitación extrema. Esto afecta la gestión del riesgo, ya que responder a los riesgos conexos al clima implica tomar decisiones en un mundo cambiante, con una incertidumbre constante acerca de la gravedad y el momento en que se sentirán los impactos del cambio climático y con límites en la eficacia de la adaptación (IPCC, 2014, p. 32).

Las figuras 3 y 4 muestran la participación en la preparación o respuesta ante los eventos extremos de precipitación. En la figura 3 se observa que el porcentaje más alto para las tres zonas es cuando la organización tiene participación antes de un evento extremo de precipitación: $10 \%$ para la zona norte, $21 \%$ para la

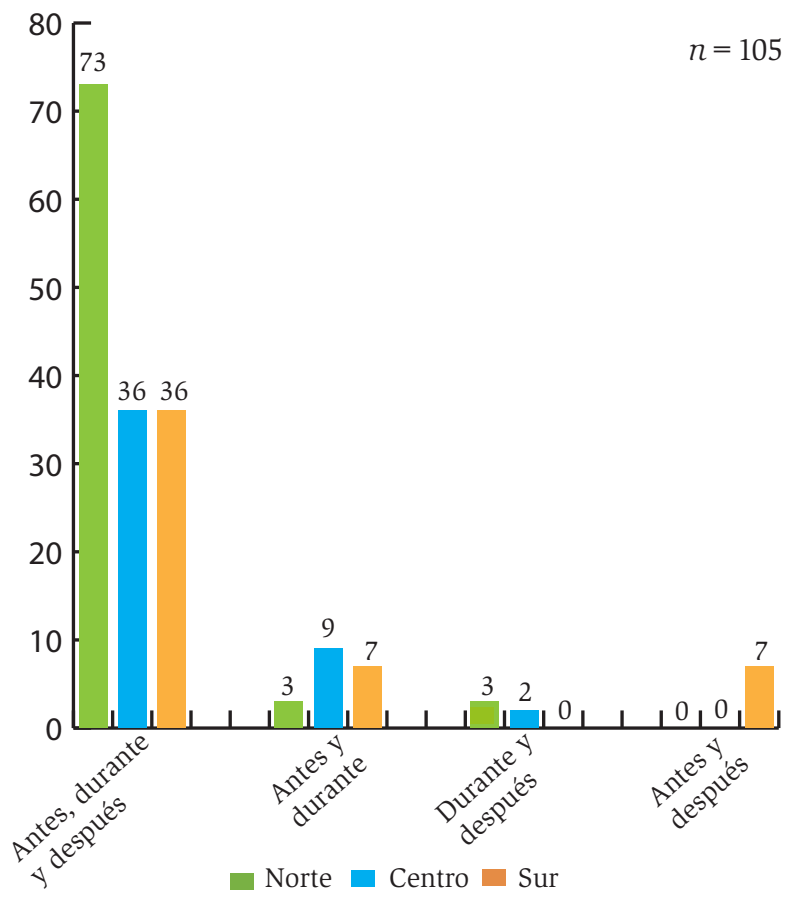

Fuente: Elaboración propia.

FIGURA 4. Porcentaje de respuestas múltiples a la pregunta: ¿Está su organización involucrada en la preparación o la respuesta a los eventos extremos de precipitación? 
centro y $32 \%$ para la sur. En los casos donde el entrevistado marcó más de una respuesta (figura 4), cerca de la mitad de los encuestados (46\%) señaló como principal respuesta la combinación antes, durante y después de un evento extremo de precipitación.

Las principales actividades que realizan los funcionarios ante una precipitación extrema son: primero, la emisión de alertas meteorológicas, mencionada por uno de cada cuatro entrevistados, sin importar la zona de procedencia; la segunda opción fue albergues, para la zona norte (40\%), y la elaboración de planes de acción, para las zonas centro (19\%) y sur (32\%); la tercera opción fue la elaboración de planes de acción, para la zona norte (18\%), asistencia pública, para la centro (17\%), y ong, para la sur (25\%) (véase figura 5).

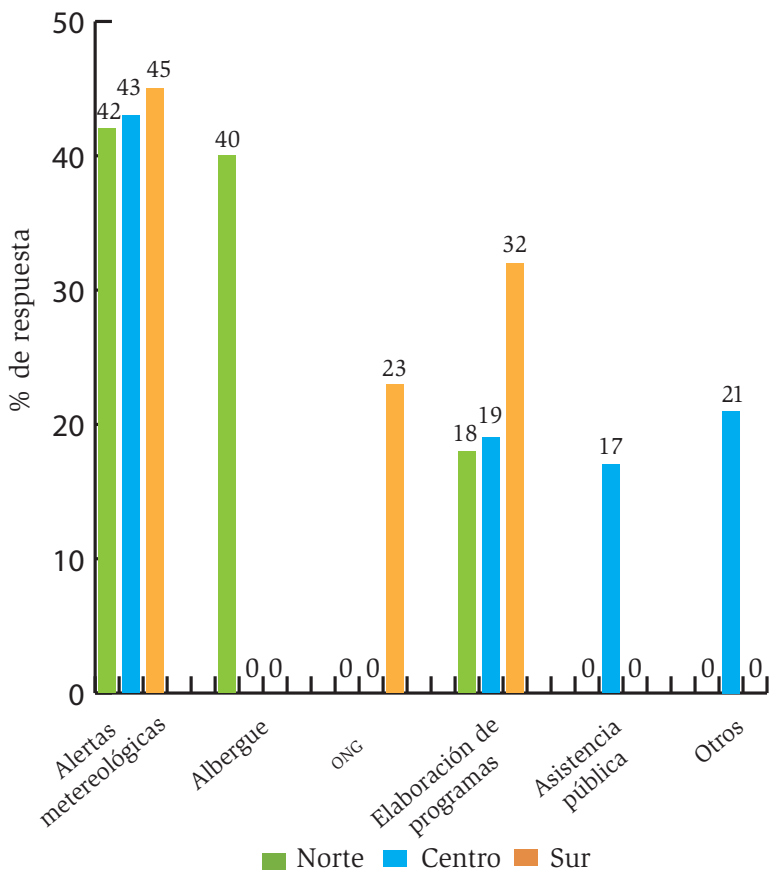

Fuente: Elaboración propia.

106 FiguRa 5. Porcentaje de respuesta a la pregunta: ¿Qué tipos de actividades describen mejor la participación de su organización ante una precipitación extrema? 
Por otra parte, cuando se les cuestionó sobre los servicios específicos que proporciona su organización con respecto a precipitaciones extremas, los entrevistados contaban con la opción de marcar todas las posibilidades, ordenándolas según su importancia. El porcentaje más alto para las tres zonas (figura 6) se registró para las alertas meteorológicas (norte $53 \%$, centro $47 \%$ y sur $32 \%$ ). Además, las principales instituciones con las que colaboran cuando se presenta una precipitación extrema son, en orden de importancia: Secretaría de Protección Civil, Secretaría de la Defensa Nacional, Secretaría de Salud, Secretaría de Marina y Cruz Roja Mexicana.

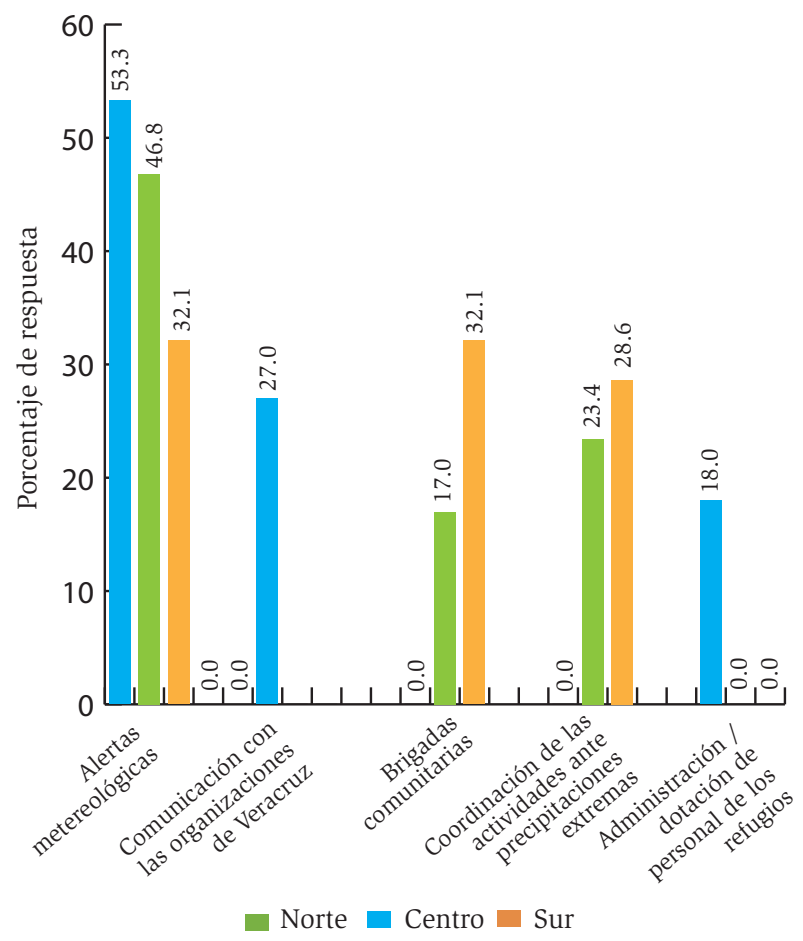

Fuente: Elaboración propia.

FIGURA 6. Porcentaje de respuesta a la pregunta: ¿Qué servicios específicos proporciona su organización con respecto a precipitaciones extremas? 
Finalmente, en la pregunta ¿Qué ayudaría a la institución a que mejore la seguridad de los usuarios ante precipitaciones extremas?, el mayor porcentaje obtenido para las zonas norte y sur está en la investigación y educación, con 56 y $37.5 \%$, respectivamente, mientras que para la zona centro fue contar con mejor infraestructura, con 33 \% (figura 7). Larsen et al. (2012, p. 12) señalan que existe una evidente falta de consenso entre las acciones efectivas de adaptación debido a las prioridades normativas y políticas.

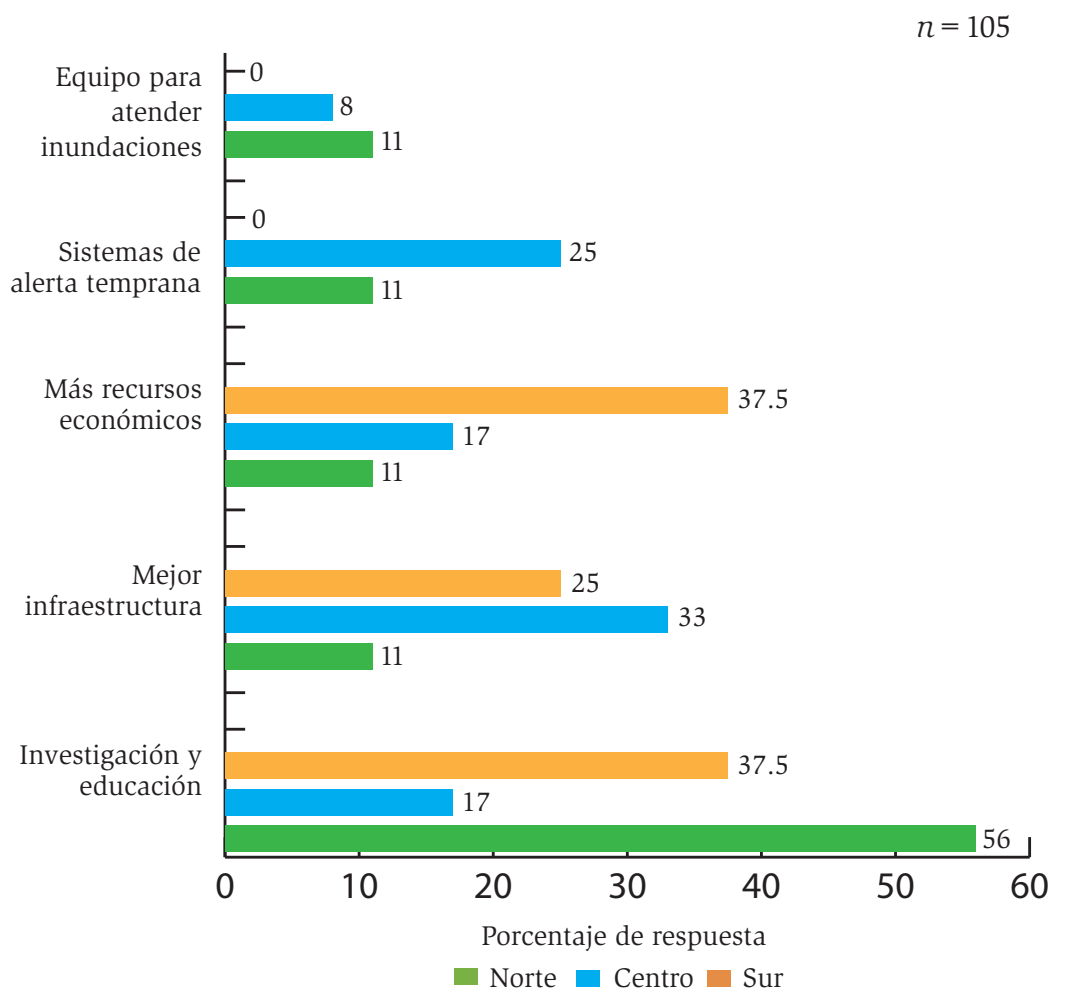

Fuente: Elaboración propia. seguridad de los usuarios ante precipitaciones extremas? 
Cabe destacar que la posibilidad de acción municipal está limitada por al menos tres aspectos: 1) las capacidades de los funcionarios municipales, que son heterogéneas; 2) los recursos escasos de los municipios, que, además, en el rubro de medio ambiente se destinan de manera prioritaria a acciones de agua, saneamiento y recolección de residuos; y 3 ) tener una gestión de tres años (actualmente cuatro para Veracruz), que es un periodo muy corto para el diseño e instrumentación de políticas de cambio climático. En este sentido, las acciones para la adaptación necesitan tener un fuerte sustento de participación social de las comunidades, a fin de garantizar su permanencia (Semarnat, 2012, p. 121).

Asimismo, entre los principios rectores que sustentan la Política Nacional de Cambio Climático (con base en la Ley General de Cambio Climático) se encuentran: a) corresponsabilidad entre el Estado y la sociedad; b) precaución ante la incertidumbre; c) prevención de los daños al medio ambiente y preservación del equilibrio ecológico; d) integralidad al adoptar un enfoque de coordinación y cooperación entre órdenes de gobierno, así como con los sectores social, público y privado; e) participación ciudadana efectiva, y f) responsabilidad ambiental. Por tanto, es relevante que se fomente un lenguaje común entre los involucrados, además de incentivar la participación social y transversalidad en la toma de decisiones ante eventos extremos de precipitación, con la finalidad de hacerla cada vez más eficiente.

Los resultados obtenidos indican que la gestión del riesgo en el estado de Veracruz es principalmente correctiva, donde los gobiernos municipales intervienen sobre el riesgo existente y tratan de reducirlo. Sin embargo, para lograr una gestión integral del riesgo se debe incorporar la gestión prospectiva y la adaptativa. La primera actúa sobre un riesgo aún no existente, para evitarlo o reducirlo. La segunda interviene sobre el riesgo no reducido o aceptado, minimizando los daños y pérdidas probables, a través de medidas que aumenten la resiliencia y la capacidad de adaptación (Gómez, 2010).

La adaptación debe realizarse en el nivel local y por ello es fundamental considerar con mayor detalle las condiciones e impactos regionales e involucrar a estados y municipios en el desarrollo de planes locales de adaptación a los eventos extremos de precipitación (Semarnat, 2013, p. 36). Además, es necesario tener en cuenta las prioridades, necesidades, conocimiento y capacidad local que empodere a las personas para planear y hacer frente a los impactos del cambio climático (Semarnat, 2013, p. 36). 


\section{Conclusiones y recomendaciones}

Derivado del análisis anteriormente presentado se concluye que la mayoría de los gobiernos municipales se involucra o participa en la preparación o respuesta a eventos extremos de precipitación antes, durante y después, pero con mayor frecuencia de forma previa. Sin embargo, por lo general se restringen a la emisión de alertas meteorológicas y a las actividades relacionadas con albergues. Por otra parte, la mayoría de los entrevistados considera un aumento en la cadena de toma de decisiones, lo que limita la eficiencia y eficacia de la gestión del riesgo.

Carter et al. (2015, p. 47) apuntan que la comunicación entre el sector público, la academia y el sector empresarial está muy poco desarrollada, como ocurre en nuestro caso, sin embargo, plantean que es una o la mejor área de oportunidad para proveer soluciones a escala local. Por lo tanto, se espera que si mejora la capacidad de entender y homogeneizar los conceptos básicos involucrados en la toma de decisiones ante los fenómenos hidrometeorológicos, también puede mejorarse la comunicación entre los niveles de gobierno, la ciudadanía y los sectores público y privado, así como incorporar nuevos conocimientos que incrementen la efectividad de la toma de decisiones.

Por tanto, se recomienda la capacitación y comunicación como estrategias esenciales para reducir la vulnerabilidad y elevar la resiliencia en materia de cambio climático, a partir de un diagnóstico de los funcionarios públicos al inicio de su gestión. Además, se deben considerar las prioridades, necesidades, conocimiento y capacidades locales, que empoderen a las personas para planear y hacer frente a los impactos del cambio climático, lo que contribuirá al establecimiento de planes de adaptación exitosos en el ámbito local. En una primera instancia permitiría la correcta comunicación entre los actores principales.

\section{Agradecimientos}

Los autores agradecen al proyecto Vulnerabilidad y Capacidad Adaptativa de los productores de Café en la región central de Veracruz, México ante los desastres hidrometeorológicos, que fue financiado por el Programa de Apoyo a Proyectos de Investigación e Innovación Tecnológica (PAPIIT), de la Universidad Nacional Autónoma de México, para el periodo 2012-2014, por las facilidades otorgadas para la realización del presente trabajo. 


\section{Fuentes consultadas}

Acevedo, F. y Luna, A. (2006). Principales fenómenos meteorológicos que afectaron al estado de Veracruz en el año 2005. En A. Tejeda Martínez (coord.), Inundaciones 2005 en el estado de Veracruz (pp. 53-67). Xalapa: Universidad Veracruzana/Consejo Veracruzano de Ciencia y Tecnología.

Armién, F. (2012). Sistemas de Alerta Temprana 10 preguntas y 10 respuestas para la comunidad educativa de Panamá. San José: Oficina San José para Centroamérica y México-Organización de las Naciones Unidas para la Educación, la Ciencia y la Cultura.

Cámara de Diputados. (6 de junio de 2012). Ley General de Protección Civil. Diario Oficial de la Federación.

Cámara de Diputados. (29 de enero de 2016). Constitución Política de los Estados Unidos Mexicanos, última reforma 29 de enero de 2016. Diario Oficial de la Federación.

Carter, J. G., Cavan, G., Connelly, A., Guy, S., Handley, J. y Kazmierczak, A. (2015). Climate change and the city: Building capacity for urban adaptation. Progress in Planning, 95, 1-66.

Fernández Bremauntz, A. (2012). Incorporación de la variable cambio climático en la gestión de recursos naturales en México. México: Banco Interamericano de Desarrollo.

Gómez, M. A. (2010). Marco conceptual bajo el cual se han desarrollado las experiencias de gestión del riesgo y adaptación al cambio climático en el Perú. En Taller Internacional Lecciones Aprendidas de la Gestión del Riesgo en Procesos de Planificación e Inversión para el Desarrollo (pp. 33-35). Lima: Ministerio de Economía y Finanzas, Perú/Gobierno Regional de Piura/Estrategia Internacional para la Reducción a Desastres (NU-EIRD)/Deutsche Gessellschaft für Technische Zusammenarbeit (GTz)/Cooperación Alemana al Desarrollo-Agencia de la Giz en el Perú. InECC. (2016). El cambio climático en México. Información por estado y sector. México: Instituto Nacional de Ecología y Cambio Climático. Recuperado de http://www2.inecc.gob.mx/cclimatico/edo_sector/esta- 
dos/futuro_veracruz.html [2016, 20 de enero].

InEgr. (2012). Perspectiva estadística. Veracruz de Ignacio de la Llave. México: Instituto Nacional de Estadística y Geografía. Recuperado de http:// www.inegi.org.mx/prod_serv/contenidos/espanol/bvinegi/ productos/integracion/estd_perspect/ver/Pers-ver.pdf [2015, 20 de octubre].

Inouye, J. (2014). Risk Perception: Theories, Strategies, and Next Steps. Campbell Institute-National Safety Council.

IPCC. (2012). Managing the Risks of Extreme Events and Disasters to Advance Climate Change Adaptation. Summary for Policymakers. In C. B. Field, V. Barros, T. F. Stocker, D. Qin, D. J. Dokken, K. L. Ebi, ... P. M. Midgley (eds.), A Special Report of Working Groups I and II of the Intergovernmental Panel on Climate Change (pp. 1-19). Cambridge: Cambridge University Press.

IPCC. (2014). Climate Change 2014: Impacts, Adaptation, and Vulnerability. Summary for Policymakers. In C. B. Field, V. R. Barros, D. J. Dokken, K. J. Mach, M. D. Mastrandrea, T. E. Bilir, ... L. L. White (eds.), Part A: Global and Sectoral Aspects. Contribution of Working Group II to the Fifth Assessment Report of the Intergovernmental Panel on Climate Change (pp. 1-32). Cambridge: Cambridge University Press.

Larsen, R. K., Swartling, A. G., Powell, N., May, B., Plummer, R., Simonsson, L. y Osbeck, M. (2012). A framework for facilitating dialogue between policy planners and local climate change adaptation professionals: Cases from Sweden, Canada and Indonesia. Environmental Science \& Policy, 23, 12-23.

Monterroso, R. A., Fernández E., A., Trejo V., R. I., Conde A., A. C., Escaldón C., J., Villers R., L. y Gay, C. (2014). Vulnerabilidad y adaptación a los efectos del cambio climático en México. México: Centro de Ciencias de la Atmósfera-Universidad Nacional Autónoma de México. Recuperado de http://atlasclimatico.unam.mx/VyA/files/assets/basic-html/ toc.html [2015, 10 de octubre].

Naciones Unidas. (2015). Convención Marco sobre el Cambio Climático. Conferencia de las Partes. $21^{\text {er }}$ período de sesiones. Aprobación del Acuerdo de París. París: Naciones Unidas. Recuperado de http://unfccc.int/ 
resource/docs/2015/cop21/spa/109s.pdf [2015, 10 de octubre].

Ochoa, C., Ibarra, S., Welsh, C. y Yáñez, G. (2015). Análisis de la precipitación extrema en zonas de montaña en Veracruz. En XXIV Congreso Mexicano de Meteorología. Organización Mexicana de Meteorólogos, A. C.

Palma, B., Morales, R. y Díaz, D. (2012). Actualización de envolventes de probabilidad empírica de impacto de ciclones en cuatro puertos del Golfo de México y a la antelación al riesgo. En A. Tejeda Martínez (coord.), Las inundaciones 2010 en Veracruz. La biosfera, escenarios y herramientas (pp. 70-89). Xalapa: Consejo Veracruzano de Investigación Científica y Desarrollo Tecnológico.

Renn, O. (1998). The role of risk perception for risk management. Reliability Engineering and System Safety, 59, 49-62.

Ruiz Barradas, A., Tejeda Martínez, A., Miranda Alonso, S. y Flores Zamudio, R. H. (2010). Climatología. En G. Benítez Badillo y C. Welsh Rodríguez (coords.), Atlas del patrimonio natural, histórico y cultural de Veracruz. Patrimonio natural (t. I, pp. 65-84). Xalapa: Comisión del Estado de Veracruz para la Conmemoración de la Independencia Nacional y la Revolución Mexicana.

Segob. (2015). Sistema de Alerta Temprana para Ciclones Tropicales. México: Sistema Nacional de Protección Civil-Secretaría de Gobernación. Recuperado de http://www.proteccioncivil.gob.mx/work/models/ProteccionCivil/Resource/62/1/images/siatctnueva.pdf [2015, 10 de octubre].

Semarnat. (2012). Adaptación al cambio climático en México: visión, elementos y criterios para la toma de decisiones. México: Instituto Nacional de Ecología y Cambio Climático-Secretaría de Medio Ambiente y Recursos Naturales.

Semarnat. (2013). Estrategia Nacional de Cambio Climático Visión 10-20-40. México: Secretaría de Medio Ambiente y Recursos Naturales.

Tejeda-Martínez, A., Guadarrama-Olivera, M. E., Ochoa-Martínez, C. A., Medina-Chena, A., Equihua Zamora, M. E., Cejudo Báez, A., ... Marín-Hernández, M. (comps.). (2008). Programa Veracruzano ante el Cambio Climático. Xalapa: Gobierno del Estado de Veracruz/Universidad Veracruzana/Embajada Británica México/Centro de Ciencias de la Atmós- 
fera-Universidad Nacional Autónoma de México/Instituto de Ecología, A. C./Instituto Nacional de Ecología-Secretaría de Medio Ambiente y Recursos Naturales.

Travieso-Bello, A. C. (2012). Aplicación del modelo Presión-Estado-ImpactoRespuesta al análisis de riesgo por inundaciones en la cuenca del río Nautla, Veracruz. En A. Tejeda Martínez (coord.), Las inundaciones 2010 en Veracruz. La biosfera, escenarios y herramientas (pp. 90-125). Xalapa: Consejo Veracruzano de Investigación Científica y Desarrollo Tecnológico.

Vázquez Torres, M., Carvajal Hernández, C. I. y Aquino Zapata, A. M. (2010). Áreas Naturales Protegidas. En G. Benítez Badillo y C. Welsh Rodríguez (coords.), Atlas del patrimonio natural, histórico y cultural de Veracruz. Patrimonio natural (t. I, pp. 249-274). Xalapa: Comisión del Estado de Veracruz para la Conmemoración de la Independencia Nacional y la Revolución Mexicana.

Welsh, C., Ochoa, C., Travieso-Bello, A. C., Morales, M., García, T., Yáñez, G. y Medina, L. (2015). Diseño y construcción de planes de acción climática en el estado de Veracruz: revisión crítica. En A. Yáñez-Arancibia (coord.), Cambio climático. Adaptación y mitigación hacia Agendas Siglo XXI (pp. 1-12). México: AGT Editor. 